\title{
Current-induced vortex dynamics in Josephson-junction arrays: Imaging experiments and model simulations
}

\author{
S. G. Lachenmann, ${ }^{*}$ T. Doderer, and R. P. Huebener \\ Lehrstuhl Experimentalphysik II, Universität Tübingen, Auf der Morgenstelle 14, D-72076 Tübingen, Germany \\ T. J. Hagenaars, ${ }^{\dagger}$ J. E. van Himbergen, and P. H. E. Tiesinga \\ Instituut voor Theoretische Fysica, Princetonplein 5, Postbus 80006, 3508 TA Utrecht, The Netherlands \\ Jorge V. José \\ Department of Physics, and Center for Interdisciplinary Research on Complex Systems, Northeastern University, 360 Huntington Avenue, \\ Boston, Massachusetts 02115 \\ (Received 13 February 1997)
}

\begin{abstract}
We study the dynamics of current-biased Josephson-junction arrays with a magnetic penetration depth $\lambda_{\perp}$ smaller than the lattice spacing. We compare the dynamics imaged by low-temperature scanning electron microscopy to the vortex dynamics obtained from model calculations based on the resistively shunted junction model, in combination with Maxwell's equations. We find three bias current regions with fundamentally different array dynamics. The first region is the subcritical region, i.e., below the array critical current $I_{c}$. The second, for currents $I$ above $I_{c}$, is a "vortex region," in which the response is determined by the vortex degrees of freedom. In this region, the dynamics is characterized by spatial domains where vortices and antivortices move across the array in opposite directions in adjacent rows and by transverse voltage fluctuations. In the third, for still higher currents, the dynamics is dominated by coherent-phase motion, and the current-voltage characteristics are linear. [S0163-1829(97)02433-8]
\end{abstract}

\section{INTRODUCTION}

Studies of Josephson-junction arrays are of interest to model vortex dynamics as well as for their application in superconducting electronics. ${ }^{1}$ Most experimental results dealing with (vortex) dynamics in two-dimensional (2D) arrays were obtained by measuring the current-voltage $(I-V)$ characteristics. Such measurements do not give the spatially resolved information needed for an unambiguous determination of the detailed microscopic dynamics underlying the measured response. By contrast low-temperature scanning electron microscopy (LTSEM) is a technique that allows for spatially resolved investigation of the dynamical states in superconducting systems. As such it offers the possibility to determine the microscopic nature of the dynamics, if one correlates the experimental information with the microscopic dynamics obtained from model calculations.

Here we present both experimental images and theoretical vortex dynamics results in dc-biased $2 \mathrm{D}$ classical arrays (i.e., with a Josephson coupling energy $E_{J}$ much larger than the charging energy $E_{c}$ ) with high damping and a small magnetic penetration depth, and in zero applied magnetic field. We gain detailed insight into the spatially resolved vortex dynamics by supplementing and comparing the LTSEM results to those obtained from model calculations employing the resistively shunted junction equations together with Maxwell's equations. Due to the small magnetic penetration depth of our samples, the applied dc current gives rise to strong induced magnetic fields at the edges of the arrays. These fields in turn facilitate the penetration of (currentinduced) vortices at the edges. In this respect there is some correspondence between inductive overdamped arrays and continuous superconducting thin films. Both systems show a current-induced resistive state due to the nucleation of vortices of opposite vorticity at opposite ends of the sample and subsequent vortex motion into the sample, as is described for superconducting bridges in Refs. 2 and 3.

We include the mutual inductances between array cells in order to take into account the self-induced fields in the model simulation. We use an algorithm developed recently in Ref. 4, which takes into account an approximate full-range inductance matrix. A particularly interesting region of array dynamics is the current region slightly above the array critical current. There we find an intricate structure in the $I-V$ curves. $^{5-8}$ Recent results of LTSEM experiments in this current region were interpreted in terms of the collective motion of current-induced vortices. ${ }^{5}$ Subsequently the detailed form of this motion was found to be in close agreement with preliminary results of numerical investigations that take into account inductive effects. ${ }^{9}$

The aim of this work is to provide insight into the array dynamics underlying the structures found in the $I-V$ curves over the whole current range. To achieve this goal we compare the experimental imaging results to graphical animations of the time evolution of the spatially resolved vortex pattern distributions obtained from our model calculations. In addition, we define and calculate a number of orderparameter-like quantities that characterize the nature of the microscopic dynamics. The main conclusion is that we can distinguish three different regions in the array dynamics: the subcritical region (I), the vortex region (II), and the region of constant differential resistance (III). In region (I) the array is in a zero-voltage state. Region (II) is dominated by (collective) vortex dynamics, contributing to the structure of the 
$I-V$ characteristics. In contrast, in region (III) the array dynamics is characterized by a "nearly coherent" behavior of the junction phases. In Sec. II we describe the samples and the imaging technique. In Sec. III we introduce the model equations and the quantities calculated. In Sec. IV the experimental measurements and the model calculation are discussed and compared. Our conclusions are presented in Sec. V.

\section{EXPERIMENTAL TECHNIQUES}

\section{A. Samples}

The samples used for the present studies consist of twodimensional arrays of $\mathrm{Nb} / \mathrm{AlO}_{x} / \mathrm{Nb}$ junctions with square elementary cells. The junctions are square with an area of about $18 \mu \mathrm{m}^{2}$. We used $6 \times 6,10 \times 10$, and $20 \times 20$ arrays without a ground plane and $10 \times 10$ and $20 \times 10$ arrays with a superconducting $\mathrm{PbIn}$ ground plane placed at a distance to the array of about $1 \mu \mathrm{m}$. Here an $N \times M$ array denotes an array that has $N$ columns of $M$ junctions. The lattice spacing $a$ is $16.7 \mu \mathrm{m}$. Each of the junctions is externally shunted by an Ohmic resistor $R_{s} \approx 1.5 \Omega$ to decrease the McCumber parameter $\beta_{c}=2 \pi i_{c} R_{s}^{2} C / \Phi_{0} \approx 0.7$ (Ref. 10) (overdamped regime), where $\Phi_{0}=h /(2 e)$ denotes the flux quantum. The critical current of each junction is $i_{c} \approx 150 \mu \mathrm{A}$. The spread of $i_{c}$ over one array is typically less than $3 \%$ (one standard deviation from the mean value). ${ }^{10}$ The magnetic penetration depth $\lambda_{\perp}=\hbar /\left(2 e \mu_{0} i_{c}\right)$ of the arrays ${ }^{11}$ is smaller than $a$, where $\mu_{0}$ is the permeability of free space and $e$ is the elementary charge. In Ref. 10 the sample geometry, layout, and fabrication are described in more detail.

\section{B. Experimental imaging of arrays}

Low-temperature scanning electron microscopy offers the possibility to image various properties of superconducting samples during their operation at liquid helium temperatures. The basic LTSEM principles together with some results are described in Refs. 12 and 13. The top surface of the sample is scanned with the electron beam, while the sample is thermally coupled to a liquid helium bath. For the present studies, the sample is dc current biased and the electron beam induces a change $\Delta V$ in the array voltage that is recorded as a function of the focus coordinates $\left(x_{0}, y_{0}\right)$ of the $e$ beam. In order to increase the sensitivity, the $e$ beam is chopped with a $20 \mathrm{kHz}$ frequency and $\Delta V$ is phase sensitively detected with a lock-in amplifier. Typical values for $\Delta V$ are in the the range $100 \mathrm{nV}$ to $5 \mu \mathrm{V}$, whereas the array voltage $V$ is of the order of $\mathrm{mV}$. Hence, the perturbation due to the $e$-beam irradiation is small. The sample temperature is estimated to be about $4.5 \mathrm{~K}$ for a helium-bath temperature of $4.2 \mathrm{~K}$. The dominant effect of the $e$-beam irradiation is local heating. We estimate from the $e$-beam parameters (electron energy 25 $\mathrm{keV}$, beam current $100 \mathrm{pA}$ ) that there is a local temperature increment at the beam focus of about $0.4 \mathrm{~K}$. The lateral extension of the thermally perturbed area is about $1 \mu \mathrm{m}<a$ (representing the limit of the spatial resolution of this imaging technique).

The sample is shielded from external magnetic fields by four $\mu$-metal shields at both room and liquid helium temperatures. A perpendicular magnetic field can be applied us- ing a circular copper coil placed in the liquid helium just below the sample substrate. In zero applied magnetic field, the residual external dc field perpendicular to the array corresponds to a frustration $f \leqq 0.1$, with $f$ the average external flux in one unit cell divided by $\Phi_{0}$. This residual magnetic field $B \approx 700 \mathrm{nT}$ was obtained by measuring the dependence of the array critical current $I_{c}$ on an applied perpendicular magnetic field for an array without a superconducting ground plane.

The experimental data shown in this paper are obtained in zero applied perpendicular magnetic field, i.e., in the residual magnetic field mentioned above. For applied fields corresponding to approximately integer values of $f$ the experimental results remain qualitatively the same up to $f \approx 5$.

To interpret our imaging results, it is important to identify the different time scales involved. The junction oscillation period is of the order of $10 \mathrm{ps}$, whereas the decay time of the beam's thermal perturbation is about $100 \mathrm{~ns} .{ }^{12}$ During stepwise scanning, the electron beam typically stays $3 \mathrm{~ms}$ at each position. The time needed to take one complete LTSEM image of the array dynamics is of the order of minutes, and hence, the beam-induced voltage signal $\Delta V\left(x_{0}, y_{0}\right)$ represents a time-averaged quantity on the time scales of Josephson dynamics.

The local temperature increment at the $e$-beam focus is most effective at the positions of the Josephson junctions. For an $e$-beam current of $100 \mathrm{pA}$, the heating of an individual junction at $\left(x_{0}, y_{0}\right)$ results in a reduction $\Delta i_{c}$ of the critical current of this junction $\Delta i_{c} / i_{c} \approx 8 \%$. The resulting voltage change $\Delta V\left(x_{0}, y_{0}\right)$ depends on the nature of the dynamics at and around the junction at $\left(x_{0}, y_{0}\right)$. Hence, a spatially inhomogeneous steady-state dynamics gives rise to an inhomogeneous image. Spatially resolved images have been interpreted in Ref. 5 in terms of vortex motion or, in the case of underdamped samples, in terms of row-switched dynamical states.

\section{MODEL EQUATIONS FOR THE DYNAMICS OF INDUCTIVE JOSEPHSON-JUNCTION ARRAYS}

In this section we briefly discuss the model we use to describe the array dynamics including the self-induced magnetic fields. In the model, the array is driven by a uniform applied dc current $I$ along the vertical direction. In the classical regime $E_{J} \gg E_{c}\left(E_{J}=\Phi_{0} i_{c} / 2 \pi\right.$ and $\left.E_{c}=e^{2} / 2 C\right)$, the phases $\theta(\mathbf{r})$ of the superconducting order parameter on an island $\mathbf{r}$ are the only variables. The array dynamics is then determined by the resistively and capacitively shunted junction (RCSJ) model for each junction and Kirchhoff's current conservation condition on the superconducting islands plus Faraday's law for the magnetic field dynamics.

Using the approximation introduced in Ref. 4 ("model C') for the full-range inductance matrix, and using the temporal gauge, one obtains a closed set of dynamical equations for the gauge-invariant phase differences $\Psi\left(\mathbf{r}, \mathbf{r}^{\prime}\right)$ $\equiv \theta(\mathbf{r})-\theta\left(\mathbf{r}^{\prime}\right)-2 \pi A\left(\mathbf{r}, \mathbf{r}^{\prime}\right)$. Here $A\left(\mathbf{r}, \mathbf{r}^{\prime}\right)$ is defined by the line integral of the vector potential $\mathbf{A}$, $A\left(\mathbf{r}, \mathbf{r}^{\prime}\right)=\left(1 / \Phi_{0}\right) \int_{\mathbf{r}^{\prime}}^{\mathbf{r}} \mathbf{A} \cdot d \mathbf{l}$. The derivation and implementation of these model equations is discussed in more detail in Refs. 4 and 14 . In the model calculations we can explicitly 


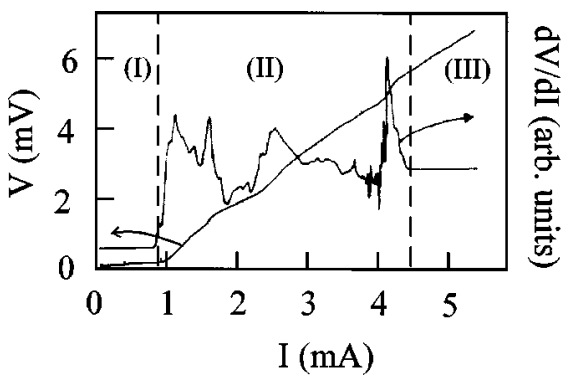

FIG. 1. $I-V$ characteristic of an experimental $I-V$ characteristic of a $10 \times 10$ array together with the differential resistance $(d V / d I)(I)$ measured at a temperature $T \approx 4.5 \mathrm{~K}$.

tune the dimensionless parameters $\beta_{c}$ and $\lambda_{\perp}$, introduced in Sec. II A.

In our simulations we obtain $\Psi(\mathbf{r}, \mathbf{r}+\mathbf{a})\left(\mathbf{a}=\mathbf{e}_{x}, \mathbf{e}_{y}\right)$ as a function of time. The magnetic flux is then given by

$$
\frac{2 \pi \Phi(\mathbf{R}, t)}{\Phi_{0}}=\sum_{\mathcal{P}(\mathbf{R})} \Psi\left(\mathbf{r}, \mathbf{r}^{\prime}, t\right) .
$$

Here $\mathcal{P}(\mathbf{R})$ is the anticlockwise sum over the four bonds $\left(\mathbf{r}, \mathbf{r}^{\prime}\right)$ around the plaquette with coordinate $\mathbf{R}$. The vorticity $n(\mathbf{R})$ is given by

$$
2 \pi\left(n(\mathbf{R})-\Phi(\mathbf{R}) / \Phi_{0}\right)=-\sum_{\mathcal{P}(\mathbf{R})} \widetilde{\Psi}\left(\mathbf{r}, \mathbf{r}^{\prime}\right),
$$

with

$$
\widetilde{\Psi}\left(\mathbf{r}, \mathbf{r}^{\prime}\right)=\Psi\left(\mathbf{r}, \mathbf{r}^{\prime}\right)-2 \pi \mathcal{N}\left(\frac{\Psi\left(\mathbf{r}, \mathbf{r}^{\prime}\right)}{2 \pi}\right),
$$

or, equivalently, by

$$
n(\mathbf{R}, t)=-\sum_{\mathcal{P}(\mathbf{R})} \mathcal{N}\left(\frac{\Psi\left(\mathbf{r}, \mathbf{r}^{\prime}\right)}{2 \pi}\right) .
$$

Here the function $\mathcal{N}$ yields the integer nearest to the argument. The voltage response for a current applied in the $y$ direction is obtained from

$$
V=\frac{1}{N M N_{t}} \sum_{\mathbf{r}} \sum_{t=1}^{N_{t}} \frac{d \Psi\left(\mathbf{r}, \mathbf{r}-\mathbf{e}_{y}, t\right)}{d t} .
$$

Here $N_{t}$ is the number of time-integration steps, and $\Sigma_{\mathbf{r}}$ is the sum over the $N \times M$ junctions in the current direction. $V$ is expressed in units of $i_{c} R_{s}$, and time is expressed in units of the dimensionless characteristic time $t_{c}=1 / \omega_{c}$ $=\hbar /\left(2 e R_{s} i_{c}\right)$.

\section{EXPERIMENTAL AND THEORETICAL RESULTS}

\section{A. Current-voltage characteristics}

Figure 1 shows a representative experimental currentvoltage $(I-V)$ characteristic together with its differential resistance $d V / d I$ obtained from a $10 \times 10$ array. The rich structure of $d V / d I$ above $I_{c}$ is typical for all arrays studied. In Fig. 1 we indicate three regions. The subcritical region (I) defined for $I<I_{c}$, an intermediate current region (II) ending at $I_{\text {lin }}$, and region (III), where the differential resistance becomes constant, for $I>I_{\text {lin }}$. In Fig. 2 we show an $I-V$ curve

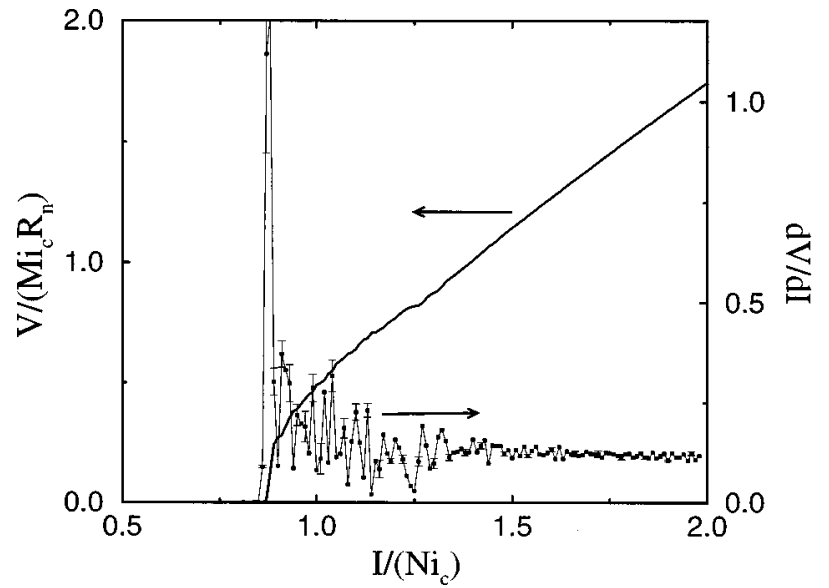

FIG. 2. Theoretical zero-temperature $I-V$ characteristics (thick line) and the differential resistance $(d V / d I)(I)$ (for selected data points we also show the error bars) of a $20 \times 20$ array with $\beta_{c}=0$ and $\lambda_{\perp}=0.6 a . V$ is the time-averaged voltage across the array in the current direction.

obtained from numerical simulation of a $20 \times 20$ array with $\beta_{c}=2 \pi i_{c} R_{s}^{2} C / \Phi_{0}=0$ and $\lambda_{\perp}=0.6 a$. Also shown is $d V / d I$ (thin line). The critical current is $I_{c} / N=0.87 i_{c}$. This $I-V$ curve is qualitatively similar to the one measured in the experiments: For intermediate currents, the differential resistance shows a jagged structure, and for currents $I \gtrsim 1.5 N i_{c}$, the $I-V$ curve is linear, just as is the case for large currents in the experimental $I-V$ curve. As our prime goal is to obtain a qualitative modeling of the experimental systems, the choice of simulation parameters is motivated partly by numerical convenience. In particular, we simulate a $\lambda_{\perp}=0.6 a$, although in experiment this ratio is about 0.1 . The latter value would lead to considerably stiffer differential equations that require much longer computation times. Due to their smaller $\lambda_{\perp} / a$ ratio, the samples studied experimentally have even stronger self-induced magnetic fields than the simulated ones, and thus smaller critical currents. Furthermore, in experiment, region (II) extends up to $I_{\text {lin }} \approx 2.9 N i_{c}$, whereas the simulations indicate that the border between regions (II) and (III) is at $I \approx 1.5 N i_{c}$.

\section{B. Imaging of array dynamics}

In this section we present images of the array dynamics for the three different regions in the $I-V$ characteristic in Fig. 1. We first discuss the subcritical region, then the region of vortex dynamics, and finally the region of constant differential resistance. Typical LTSEM imaging results are shown in Fig. 3 and model simulation results in Fig. 4.

\section{Subcritical region $\left(I<I_{c}\right)$}

For a bias current $I<I_{c}$ and in the absence of LTSEM heating, the array is in the zero-voltage state. Nevertheless, we obtain useful information from the dynamical imaging experiments. In particular, we will see below that the LTSEM images obtained in this region confirm the importance of inductive effects in our small- $\lambda_{\perp}$ samples.

In Fig. 3(a) we show a typical LTSEM imaging result below but close to the array critical current $I_{c}$, for relatively high beam power. From this result we see that junctions at or 

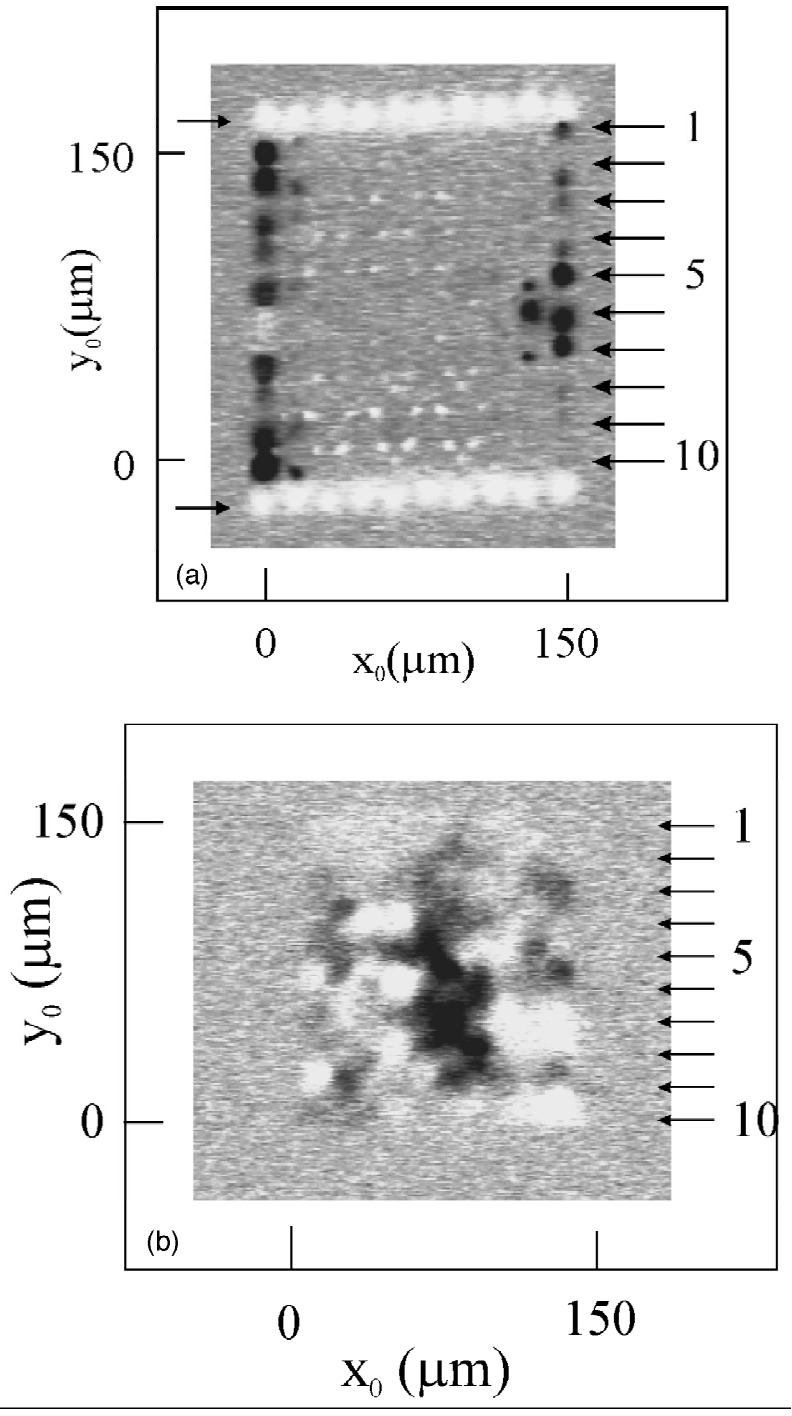

FIG. 3. Grey value representations of the experimental voltage image $\Delta V\left(x_{0}, y_{0}\right)$ for a $10 \times 10$ array at $T \approx 4.5 \mathrm{~K}$. The array is current biased at $I=0.9 I_{c}$ (a) and $I=4.25 I_{c}$ (b), respectively. The dc bias current flows vertically through the array. The array boundaries lie between $0 \mu \mathrm{m}$ and $150 \mu \mathrm{m}$ in both directions. A positive (negative) $e$-beam-induced voltage signal $\Delta V\left(x_{0}, y_{0}\right)$ is indicated by the dark (bright) areas, whereas zero signal is shown by the area surrounding the array. The individual rows of junctions are indicated by the small arrows numbered 1-10 from top to bottom. In (a), the $\Delta V<0$ voltage response at the top and bottom of the array columns (marked by arrows from the left) arises from the current feeding resistors made from InAu thin films.

near the edges parallel to the bias current give a voltage response to the local heating. This can be understood in the following way. For currents in this subcritical region, but close to the array critical current $I_{c}$, the LTSEM is acting as an active probe, inducing vortex motion. When the $i_{c}$ of a junction at the array edge is lowered due to the $e$-beam irradiation, vortices can overcome the energy barrier for entry at this junction and subsequently travel across the array. As a result, a voltage signal $\Delta V>0$ is observed. The corresponding process for junctions not at the edges is the creation of a vortex-antivortex pair. From Fig. 3(a) we see that this latter process does not occur for junctions that are not close to the edges. This means that such junctions carry less current, which corresponds to larger energy barriers for vortexantivortex creation. Such an inhomogeneous distribution of the bias current is in agreement with the small magnetic penetration depth $\lambda$ of the array. The correspondingly strong inductive effects lead to a spatial distribution of the dc bias current that is strongly peaked at the two array edges parallel to the current flow. ${ }^{15,16}$ The current-induced flux is also maximal at these edges. It is oriented in opposite directions at opposite edges. This is illustrated in Fig. 5, where we plot the numerically calculated current and magnetic field distributions for a $20 \times 20$ array at $I=0.86 N i_{c}$ and with $\lambda_{\perp}=0.6 a$. Similar simulation results in this subcritical region have been reported in Refs. 14 and 15.

\section{Vortex dynamics region $\left(I_{c}<I<I_{l i n}\right)$}

Above $I_{c}$ the current through the edge junctions exceeds the junction critical current. As a result, vortices enter the array at one edge and antivortices at the opposite edge. These vortices are depinned from the edges by the Lorentz force and move across the array, generating the observed voltage.

LTSEM results for bias currents slightly above $I_{c}$ are presented and discussed in Refs. 5-8. In the LTSEM images obtained in this current region it is seen (see, e.g., Ref. 5) that the sign of the voltage signal near the sample edges tends to alternate along the current direction. These images indicate an alternating or staggered crossing vortex motion where vortices and antivortices are nucleated at opposite array edges and subsequently move across the whole array. At the array edge opposite to the nucleation site they leave the array or, equivalently, annihilate with an image vortex of opposite sign. We will see below that this interpretation is confirmed by the general behavior found in simulation images for a bias current slightly above the array critical current. This kind of alternating crossing vortex motion has no observed analog in continuous superconducting samples.

To analyze the dynamics of the vortices in full detail, we have studied graphical animations of the time evolution of the vortex distributions in the array. First we discuss the ones obtained in a simulation for $I=0.95 N i_{c}$ of a $20 \times 20$ array with $\beta_{c}=0$ and $\lambda_{\perp}=0.6 a$. When we start the simulation with random initial phases we observe, after a transient of about $t / t_{c}=800$ time units, the type of vortex motion depicted in Fig. 4(a). The snapshots in Fig. 4(a) show the same type of vortex motion as the one deduced from the LTSEM measurements: Vortices of opposite sign cross the array in opposite directions in adjacent rows. We observe that the staggered structure is broken at two places, where two adjacent rows are crossed by vortices of equal sign. One might view these places as domain wall defects between two different polarities of the staggered pattern (for experimental results see also Ref. 5). We have verified that this type of dynamics is stable for very long simulation times. The position and number of domain walls depend sensitively on the initial conditions. For all currents in the range $I_{c} /\left(N i_{c}\right)=0.87 \leqslant I /\left(N i_{c}\right) \leqslant 1.15$ the long-time stable vortex dynamics is of the same staggered type. The dynamical patterns observed experimentally as well as numerically for $I \gtrsim I_{c}$ are strongly influenced by vortex-vortex interactions. These interactions lead to the almost regular patterns in which the vortices tend to move. Towards the high-current end of region (II), the dynamics is different. Imaging the 
(a)

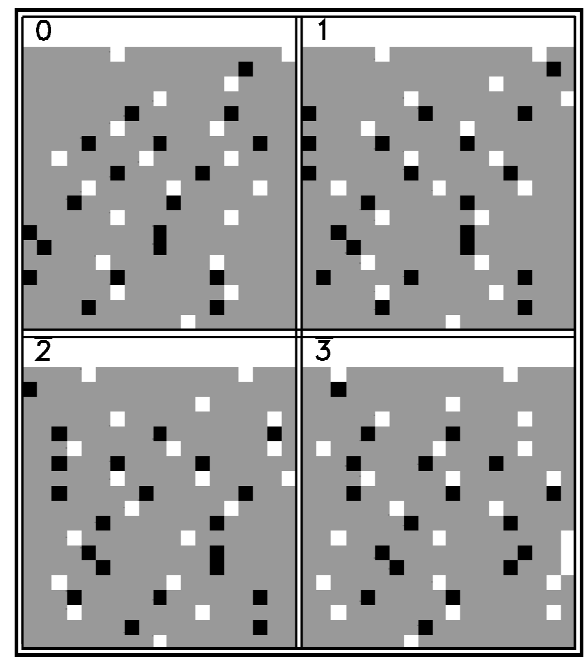

(b)

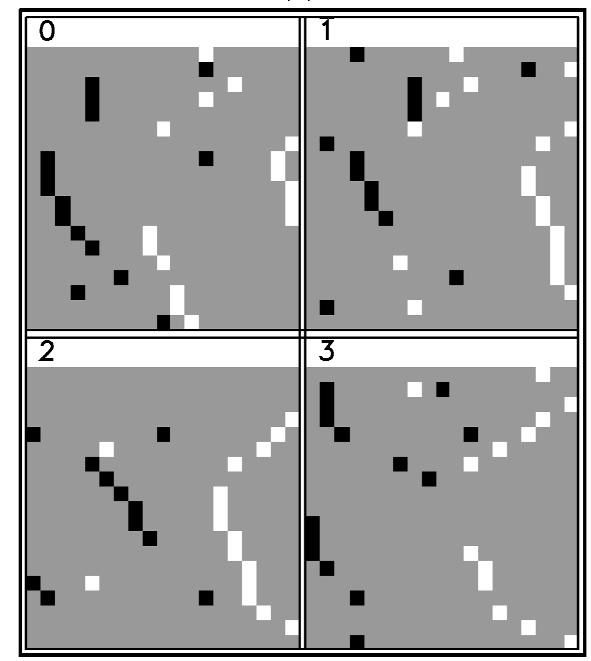

(c)

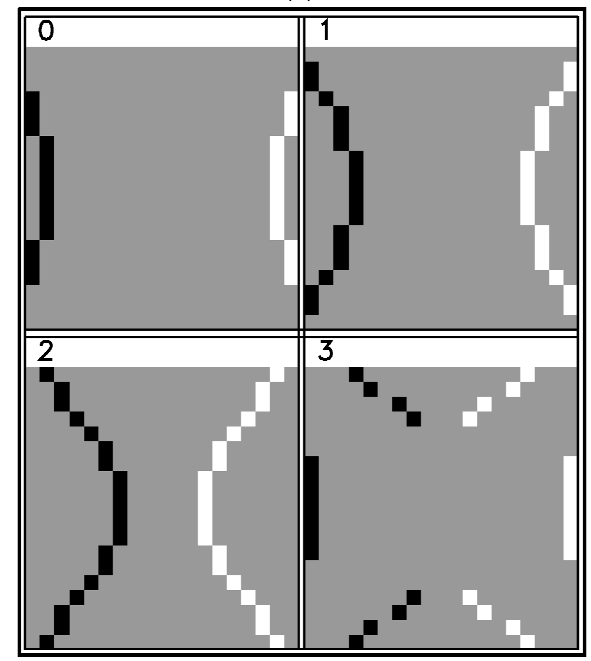

FIG. 4. Vortex configurations for simulations of a $20 \times 20$ array, for three different values of the bias current (a) - (c). For each current value, we show four consecutive frames (0-3). The dc bias current flows vertically through the array. The black (white) squares denote plaquettes with vortex number $n(\mathbf{R})=+1(-1)$. Vortices with positive sign move to the right, vortices with negative sign to the left, as can be deduced by comparing consecutive frames. (a) $I=0.95 N i_{c}$. Frame 0 is at $t=3000 t_{c}$. Between consecutive frames there is a time interval of $3 t_{c}$. Adjacent rows tend to be crossed by vortices of opposite sign. The alternating structure is disrupted between the third and fourth rows from above and between the sixth and seventh rows from below. (b) $I=1.20 N i_{c}$. Frame 0 is at $t=9025 t_{c}$. Between consecutive frames there is a time interval of $2 t_{c}$. (c) $I=2.0 N i_{c}$. Frame 0 is at $t=500.75 t_{c}$. Between consecutive frames there is a time interval of $0.9 t_{c}$.

dynamical state by LTSEM now yields larger twodimensional domain patterns spreading over several unit cells in both the $x$ and $y$ directions. A typical example for this kind of voltage response is given in Fig. 3(b). From our experimental observations, we can deduce the following: (1) The dynamical state, which gives rise to the voltage image, is stable in time. If the parameters of the sample $(I, T)$ are not changed, a subsequent image will give the same result. (2) The imaging results depend sensitively on the history. If, for example, the bias current or temperature is changed significantly and then returned to the same parameter values, the imaging results change. (3) When $I$ or $T$ is changed smoothly, we observe a smooth variation of the detected patterns. (4) A magnetic field (noninteger $f$ or $|f|>5$ ) changes the regular pattern, observed for small bias currents discussed above, to a complex response similar to that shown in Fig. 3(b). Based on these observations, we conclude that the voltage response is caused by a complex multivortex dynamics, and not by, e.g., sample inhomogeneities, trapped flux, or temperature fluctuations.

In our simulations, the staggered vortex dynamics is the relevant dynamics up to approximately $I=1.15 N i_{c}$. The region $1.15 \leqq I /\left(N i_{c}\right) \lesssim 1.5$ is a transition region between the regime of staggered vortex dynamics and the regime of constant differential resistance. In this current interval, in some parts of the array vortices move independently, and in others we observe vortices that tend to move coherently in adjacent rows. A representative example of this type of dynamics is given in Fig. 4(b).

\section{Linear branch $\left(I>I_{l i n}\right)$}

In region (III), where the $I-V$ curve is linear (constant differential resistance), each junction in the array columns (longitudinal junction) yields approximately the same volt- age signal $\Delta V\left(x_{0}, y_{0}\right)$ and the LTSEM image is rather uniform over the whole array. For example, at $I=5 I_{c}$ for a $10 \times 10$ array the voltage signal at each junction was the same within $5 \%$ (see Fig. 7 of Ref. 8).

The absence of any structure in the beam-induced voltage signal suggests that there are no isolated vortices entering or leaving the array. This is indeed what we find in the simulations in the region of constant differential resistance. In fact, the dynamics in this current region is due to a state in which the phases of the longitudinal junctions belonging to the same column oscillate almost in phase. This phase coherence reveals itself in a wavelike dynamics of the magnetic field distribution. When looking at the discrete vortex configurations shown in Fig. 4(c), we observe fronts of vortices that move inward from the boundaries. In the middle, the vortices annihilate. The discrete vortex configurations in Fig. 4(c) show a high degree of symmetry, which reflects the coherence in the motion of the longitudinal junctions in different rows. We have also explicitly simulated a scanning-induced $8 \%$ critical-current reduction of subsequent individual junctions for $I=2.0 N i_{c}$, and indeed find a spatially uniform voltage change.

\section{Crossover from vortex-dominated to coherent-phase dynamics}

In the above we have focused on comparing the LTSEM images with snapshots of vortex configurations from numerical simulations. We found three regions in the $I-V$ characteristics as well as in the experimental and numerically obtained images. We related these regions to different types of dynamics. The crossover from a vortex-dominated to a coherent-phase dynamics can be explored in more detail in the model simulations. To this end, we can consider a number of quantities that probe the degree of vortex organization 


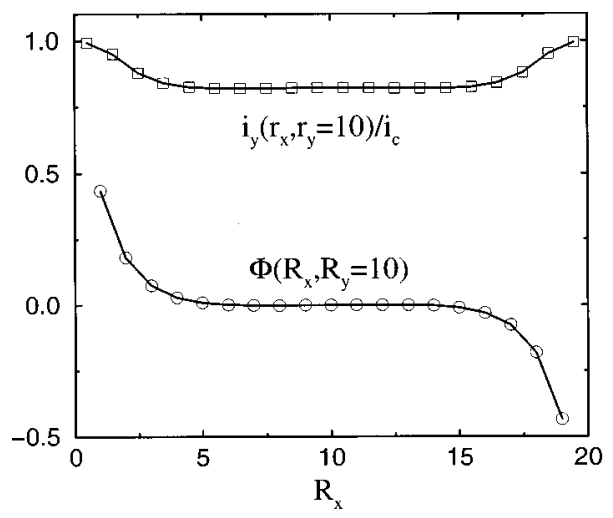

FIG. 5. Simulated field and current distribution in the central row of a $20 \times 20$ array with $\lambda_{\perp}=0.6 a$ and $I=0.86 N i_{c}$, just below the array critical current. $i_{y}$ is the current through a longitudinal junction.

or the degree of phase coherence. In particular, we find that the following order-parameter-like quantities $P$ and $P_{s}$ can be used to distinguish the different current ranges:

$$
\begin{gathered}
P \equiv\left\langle\frac{\sum_{Y=1}^{M}\left|n_{c}(Y, t)\right|}{N_{V}(t)}\right\rangle, \\
P_{s} \equiv\left\langle\frac{\sum_{Y=1}^{M}(-1)^{Y} n_{c}(Y, t)}{N_{V}(t)}\right\rangle,
\end{gathered}
$$

where

$$
n_{c}(Y, t) \equiv \sum_{X=1}^{N-1} n(\mathbf{R}, t), \quad N_{V}(t) \equiv \sum_{\mathbf{R}}|n(\mathbf{R}, t)| .
$$

$X$ and $Y$ denote the $x$ and $y$ components of the plaquette coordinate $R$, respectively: $\mathbf{R}=X \mathbf{e}_{x}+Y \mathbf{e}_{y}$. The physical meaning of $P$ and $P_{s}$ can be inferred from the fact that a vortex that enters at one side of the array either leaves the array on the other side or is annihilated by an antivortex moving in the opposite direction. The quantity $n_{c}(Y, t)$ for row $Y$ distinguishes between these two possibilities. In the former case $n_{c}$ is nonzero, whereas in the latter case it is, on average, zero. A value of $P=1$ thus implies that all the vortices cross the whole array unobstructed, while $P=0$ implies that the vortices are annihilated in the middle of the array. The staggered order parameter $P_{s}$ measures whether the spatially resolved vortex dynamics consists of alternating rows of vortices and antivortices crossing the array. The presence of domain walls, i.e., two adjacent rows in which vortices cross the array in the same direction, reduces $P_{s}$.

On the basis of this interpretation we expect that Fig. 4(a) [low-current region (II)] corresponds to $P \approx 1, P_{s} \approx 1$, and Fig. 4(c) [region (III)] to $P \approx 0$ and $P_{s} \approx 0$. In Fig. 6 we plot $P$ and $P_{s}$ versus the applied current. Indeed, directly after depinning, $I>I_{c} \approx 0.87 N i_{c}, P$ and $P_{s}$ attain values close to 1 . As the current increases this value slowly decreases [lowcurrent region (II)]. At $I \approx 1.2 N i_{c}$ the value of both order parameters exhibits a sharp drop to a much smaller (but nonzero) value. In this region the order parameters slowly decrease to the values near zero in (III). Thus, using $P$ and $P_{s}$ one can readily establish the type of vortex dynamics without having to study vortex animations for each value of the bias (a)

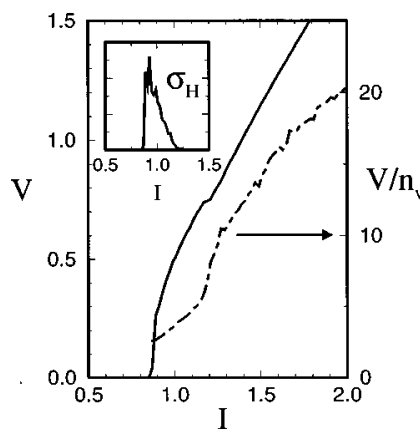

(b)

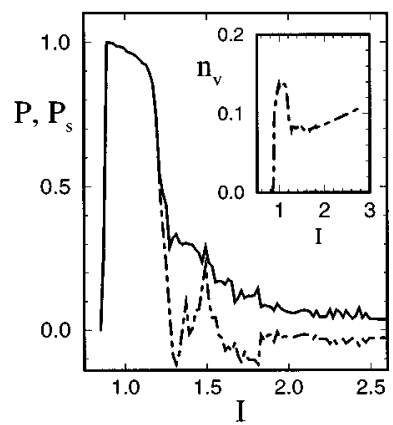

FIG. 6. Crossover from vortex-dominated to coherent-phase dynamics as a function of applied current $I$, probed by (a) $V$ (solid line), $V / n_{v}$ (dot-dashed line), and the variance $\sigma_{H}$ of the Hall voltage (inset, in arbitrary units); (b) $P$ (solid line), $P_{s}$ (dot-dashed line), and $n_{v}$ (inset). All results are obtained in an upward current sweep of a $20 \times 20$ array for $\kappa=0.6$ and $T=0$. For each current value we used a warm-up time of $1000 t_{c}$ and an averaging time of $2000 t_{c}$.

current. In particular, long-time-stable values $P \approx 1, P_{s} \approx 1$ correspond to a staggered crossing vortex motion.

We will now correlate the different regions, mapped out using $P$ and $P_{s}$, with the behavior of other quantities. In Fig. 6 (a) we show both the voltage and the average velocity per vortex $V_{v}=V / n_{v}$ (the voltage normalized by the number of vortices) versus current. In the inset of Fig. 6(b) we plot the vortex density. We observe that the vortex density displays a pronounced maximum in region (II), accompanied by a smaller slope of the $V_{v}(I)$ curve as compared to the other regions. The dynamical properties of the vortices in this region are thus different from the other regions.

Vortex jumps across a junction not only give rise to a contribution to the longitudinal voltage across the array, but also induce a fluctuation of the transverse (Hall) voltage around zero. For the nearly antisymmetric dynamical patterns of vortices in the phase-coherence region (III), like the ones shown in Fig. 4(c), the Hall voltage contributions of the left and right halves of the array tend to cancel by symmetry. In the region with staggered crossing vortex motion, however, the vortex jumps in the left half of the array do not occur in unison with antivortex jumps in the right half, leading to larger fluctuations in the Hall voltage. Therefore the Hall voltage fluctuations may be viewed as a measure of the degree of (anti)symmetry in the dynamics. Indeed, as seen in the inset of Fig. 6(a), the magnitude of these fluctuations is reduced dramatically between the vortex-dominated and the coherent-phase regime.

Figure 6(a) is obtained in an upward current sweep. We have also performed a downward current sweep from region (III), starting with a uniform phase configuration. We find that region (III) is exactly characterized by $P=P_{s}=0$, whereas in the upward sweep there are still some asymmetries in the vortex configuration that yield small but nonzero values for the order parameters. Entering the high-current end of (II), $P$ and $P_{s}$ attain nonzero values. There is a slight hysteresis in the current value at which $P$ and $P_{s}$ attain values close to 1 .

The power spectrum of the voltage, 


$$
S(\nu)=\left|\int d t V(t) e^{i 2 \pi \nu t}\right|^{2},
$$

is another useful probe for the vortex dynamics. A nonzero voltage can be viewed as either created by a vortex jump or by a junction phase slip. When the longitudinal junctions oscillate coherently, $S(\nu)$ consists of sharp peaks at multiples of $\nu=V /\left(2 \pi t_{c}\right)$. If the dynamics, however, is dominated by incoherent vortex jumps, the peaks in the spectrum are much broader. In Fig. 7 we show $S(\nu)$ for three different currents. From $i_{b}=0.95$, within the "vortex" region, to $i=2.0$ [region (III)] the spectrum changes from a noisy to sharply peaked one. These results again illustrate the crossover from a vortex-dominated dynamics to a coherent-phase dynamics for higher currents.

\section{CONCLUSIONS AND DISCUSSION}

In summary, we have shown that the comparison of LTSEM images and results of model calculations can significantly increase our insight in the dynamics of Josephsonjunction arrays. On the one hand, the comparison of the experimental images with the results of model simulations corroborates the interpretation of images for currents not too far from the array critical current, and contributes to our understanding of the dynamics underlying the images outside this current region. It shows that the dynamics we found exists even in the absence of the disturbance produced by the measuring device. On the other hand, the agreement with the experimental results supports the relevance of the model equations employed. In the context of the samples studied here, an essential ingredient of these model equations is the inclusion of (strong) self-induced magnetic fields.

The successful comparison between experimental and theoretical results has enabled us to map out three regions with different types of dynamics for dc-biased arrays with $\lambda_{\perp}<a$ in zero applied magnetic field. For bias currents above the array critical current we found current-induced vortex nucleation at the array edges parallel to the bias current. We identified an alternating pattern of crossing vortices and antivortices as the typical vortex dynamics existing for bias currents slightly above the array critical current. We conclude that at least part of the rich structure found in the experimental $I$ $V$ 's is due to the dynamics of vortices. ${ }^{17,18}$ For larger currents, the $I-V$ characteristic becomes linear, and the underlying dynamics is characterized by a growing tendency of longitudinal junctions to oscillate in phase. We have further illustrated the crossover from vortex-dominated to coherentphase-like dynamics by numerically studying the spectral function, the Hall-voltage fluctuations, and order-parameterlike quantities.

Recently Oppenländer and co-workers ${ }^{19}$ have also calculated $I-V$ characteristics similar to the experimental ones, however, without taking into account the dynamics of the self-induced magnetic fields that are essential for a successful theoretical description of the experimentally observed microscopic dynamics. In their work the structure in the differential resistance is due to the inclusion of strong (magnetic) disorder. ${ }^{20}$

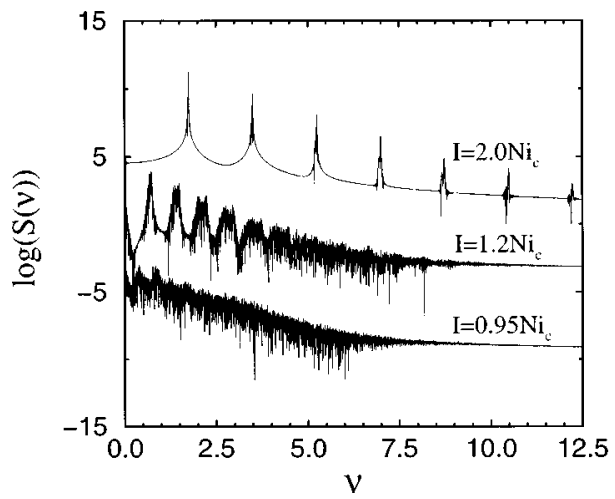

FIG. 7. Calculated power spectrum of the array voltage for three different bias currents. Subsequent curves are offset by 5.5 units.

In this paper, we have discussed the numerical results for a zero McCumber parameter. In the experiments, the McCumber parameter was estimated to be $\beta_{c}=0.7$. In our model simulations, the microscopic dynamics for currents above but close to the array critical current remains qualitatively the same for this value of the McCumber parameter. For higher currents the dynamics is again characterized by a large degree of spatial coherence. The region of genuine vortex dynamics shrinks with increasing $\beta_{c}$. For $\beta_{c} \geqslant 2.5$ the system enters a row-switched state immediately above the critical current (for $\lambda_{\perp}=0.6 a$ ), and therefore no vortex-flow regime is found. For nonzero applied magnetic field, rowswitched states in inductive arrays were studied in Ref. 21.

For the arrays used in the present studies, the KosterlitzThouless-Berezinskii phase transition temperature $T_{\mathrm{KTB}}$ is close to the superconducting transition temperature $T_{c}$ of the $\mathrm{Nb}$ thin films. The experiments are performed at temperatures well below $T_{\mathrm{KTB}}, 2 e k_{B} T / \hbar i_{c} \sim 1 \times 10^{-3}$. For these temperatures the effect of thermally induced vortices on the array dynamics is negligible. ${ }^{22}$ We have also performed calculations for $\lambda_{\perp} \geq a$ (outside the region of the present experiments) up to $\lambda_{\perp}=10 a{ }^{23}$ In this regime we have found a staggered vortex dynamics similar to the one observed for $\lambda_{\perp} \leq a$, again occurring for currents slightly above the array critical current. This indicates that the staggered vortex dynamics is the generic dynamics for such currents.

In the LTSEM experiments, the images corresponding to the staggered vortex dynamics were found for some range of magnetic frustrations around zero. We have simulated the dynamics in the lower part of region (II) (where the alternating crossing vortex motion is observed) in a magnetic frustration of $f=0.01$. For currents slightly above $I_{c}$, we find that the array evolves towards a similar state as found for $f=0$, but now there are on average more vortices with positive vorticity than with negative vorticity.

\section{ACKNOWLEDGMENTS}

We appreciate fabrication of the samples by P. A. A. Booi and S. P. Benz, National Institute of Standards and Technology, Boulder, CO. We thank G. Filatrella, C. Giovannella, W. Güttinger, F. Hilbert, D. Hoffmann, M. Keck, A. Laub, J. Oppenländer, T. Träuble, and A. V. Ustinov for valuable discussions. This work was supported by the EU Human 
Capital and Mobility program (Contract No. CHRX-CT920068) and the Bundesminister für Bildung, Wissenschaft, Forschung, und Technologie under Grant No. 13N6436. This work has been partially supported by NSF Grant No. DMR-
9521845 (J.V.J.), by the Dutch Organisation for Fundamental Research (FOM) (P.T. and J.E.H.), and by the Bayerischer Forschungsverbund Hochtemperatur-Supraleiter (FORSUPRA) (T.J.H.).
*Present address: Institut für Schicht- und Ionentechnik, Forschungszentrum Jülich, D-52425 Jülich, Germany.

†Present address: Institut für Theoretische Physik, Universität Würzburg, Am Hubland, D-97074 Würzburg, Germany.

${ }^{*}$ Present address: Center for Interdisciplinary Research on Complex Systems, Northeastern University, 360 Huntington Avenue, Boston, Massachusetts 02115.

${ }^{1}$ For a recent review of the field see, e.g., Proceedings of the ICTP workshop on Josephson junction arrays, Trieste, 1995, edited by H. A. Cerdeira and S. R. Shenoy [ Physica B 222, 253 (1996)]; Macroscopic Quantum Phenomena and Coherence in Superconducting Networks, edited by C. Giovanella and M. Tinkham (World Scientific, Singapore, 1995).

${ }^{2}$ L. G. Aslamazov and A. I. Larkin, Zh. Éksp. Teor. Fiz. 68, 766 (1975) [Sov. Phys. JETP 41, 381 (1975)].

${ }^{3}$ R. P. Huebener, Magnetic Flux Structures in Superconductors (Springer, Berlin, 1979).

${ }^{4}$ D. Domínguez and J. V. José, Int. J. Mod. Phys. B 8, 3749 (1994).

${ }^{5}$ S. G. Lachenmann, T. Doderer, D. Hoffmann, R. P. Huebener, P. A. A. Booi, and S. P. Benz, Phys. Rev. B 50, 3158 (1994).

${ }^{6}$ S. G. Lachenmann, T. Doderer, R. P. Huebener, P. A. A. Booi, and S. P. Benz, in Nonlinear Superconducting Devices and

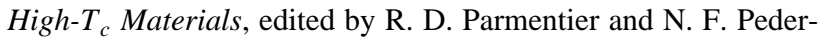
sen (World Scientific, Singapore, 1995), p. 365.

${ }^{7}$ T. Doderer, S. G. Lachenmann, R. P. Huebener, P. A. A. Booi, and S. P. Benz, IEEE Trans. Appl. Supercond. AS-5, 2723 (1995).

${ }^{8}$ T. Doderer, S. G. Lachenmann, and R. P. Huebener, in Macroscopic Quantum Phenomena and Coherence in Superconducting Networks, edited by C. Giovanella and M. Tinkham (World Scientific, Singapore, 1995), p. 317.

${ }^{9}$ T. J. Hagenaars, J. E. van Himbergen, P. H. E. Tiesinga, J. V. José, and S. G. Lachenmann, in Macroscopic Quantum Phenomena and Coherence in Superconducting Networks, edited by C. Giovanella and M. Tinkham (World Scientific, Singapore, 1995), p. 329.

${ }^{10}$ S. P. Benz and C. J. Burroughs, Appl. Phys. Lett. 58, 2162 (1991); Supercond. Sci. Technol. 4, 561 (1991).
${ }^{11}$ K. K. Likharev, Dynamics of Josephson Junctions and Circuits (Gordon and Breach, Philadelphia, 1986).

${ }^{12}$ R. P. Huebener, in Advances in Electronics and Electron Physics, edited by P. W. Hawkes (Academic Press, New York, 1988), p. 1.

${ }^{13}$ R. Gross and D. Koelle, Rep. Prog. Phys. 57, 651 (1994).

${ }^{14}$ D. Domínguez and J. V. José, Phys. Rev. B 53, 11692 (1996).

${ }^{15}$ J. R. Phillips, H. S. J. van der Zant, J. White, and T. P. Orlando, Phys. Rev. B 50, 9387 (1994).

${ }^{16}$ F. Busse, P. Seidel, and M. Darula, in Weak Superconductivity, edited by Š. Beňačka, P. Seidel, and V. Štrbík (Department of Cryoelectronics, Institute of Electrical Engineering, Slovak Academy of Sciences, Bratislava, 1994), p. 146.

${ }^{17}$ Booi and Benz Ref. (24) attributed part of the structure in region (II) to resonances between the junction capacitance and a combination of the inductances of the shunt resistors and the array cell.

${ }^{18}$ In the context of superconducting bridges, several authors (see, for example, Refs. 25 and 26) have reported structures in the $I$ $V$ characteristic and attributed those to viscous vortex motion.

${ }^{19}$ J. Oppenländer, G. Dangelmayr, and W. Güttinger, Phys. Rev. B 54, 1213 (1996).

${ }^{20}$ In Ref. 19 it is assumed that there are no transverse voltage fluctuations; i.e., the supercurrents in the transverse direction are held constant. As a result, the array dynamics decouples into $N$ independent one-dimensional, nonuniform, and nonlinear chain equations for each column. The nontrivial nature of the couplings of the two-dimensional system is retained in the randomly chosen, time-independent phase differences in the transverse direction, which one may interpret in terms of the presence of a (strong) static random magnetic field.

${ }^{21}$ J. R. Phillips, H. S. J. van der Zant, and T. P. Orlando, Phys. Rev. B 50, 9380 (1994).

${ }^{22}$ P.H.E. Tiesinga, Ph.D. thesis, Universiteit Utrecht, 1996.

${ }^{23}$ T. J. Hagenaars et al. (unpublished).

${ }^{24}$ P. A. A. Booi and S. P. Benz, IEEE Trans. Appl. Supercond. AS-5, 2899 (1995).

${ }^{25}$ R. P. Huebener and H. L. Watson, Phys. Rev. B 9, 3725 (1974).

${ }^{26}$ M. V. Pedyash, G. J. Gerritsma, D. H. A. Blank, and H. Rogalla, IEEE Trans. Appl. Supercond. AS-5, 1387 (1995). 